Kummer, J. A., E. M. Bayne, and C. S. Machtans. 2016. Comparing the results of recall surveys and standardized searches in understanding birdwindow collisions at houses. Avian Conservation and Ecology 11(1):4. http://dx.doi.org/10.5751/ACE-00820-110104

Copyright (C) 2016 by the author(s). Published here under license by the Resilience Alliance.

Research Paper

\title{
Comparing the results of recall surveys and standardized searches in understanding bird-window collisions at houses
}

\author{
Justine A. Kummer ${ }^{1}$, Erin M. Bayne ${ }^{1}$ and Craig S. Machtans ${ }^{2}$ \\ ${ }^{1}$ Department of Biological Sciences, University of Alberta, ${ }^{2}$ Environment Canada, Canadian Wildlife Service
}

\begin{abstract}
Every year a large number of birds die when they collide with windows. The actual number is difficult to ascertain. Previous attempts to estimate bird-window collision rates in Canada relied heavily on a prior citizen-science study that used memorybased surveys. Such an approach to data collection has many potential biases. We built upon this study and its recommendations for future research by creating a citizen-science program that actively searched for collision evidence at houses and apartments for an extended period with the objective to see how standardized approaches to data collection compared with memory recall. Absolute collision estimates as well as relative differences were compared between residence types in the two studies, and we found considerable differences in absolute values for collisions but similar rankings of collision rates between residence types. Collision recall rates in our study $(56.5 \%)$ were very similar those in the prior 2012 study, where $50.5 \%$ of participants remembered a bird colliding with a window at some time in the past. Fatality estimates, however, were 1.4 times higher in the 2012 study than in our study based on standardized searches. Rural houses with a bird feeder consistently had the highest number of collisions. This suggests that memory recall surveys may be a useful tool for understanding the relative importance of different risk factors causing bird-window collisions.
\end{abstract}

\section{Comparaison des résultats de relevés fondés sur le souvenir et de recherches standardisées dans la compréhension des collisions d'oiseaux avec les fenêtres de maisons}

RÉSUMÉ. Chaque année, un grand nombre d'oiseaux meurent à la suite d'une collision avec une fenêtre. Or, ce nombre d'oiseaux morts est difficile à établir. Au Canada, les essais antérieurs destinés à estimer le taux de collisions d'oiseaux avec les fenêtres reposaient grandement sur une étude à participation citoyenne qui utilisait des relevés fondés sur le souvenir. Ce genre d'approche pour récolter des données comporte de nombreux biais potentiels. À partir de cette étude et de ses recommandations pour des recherches futures, nous avons créé un programme de science citoyenne fondé sur une recherche active d'indices de collisions avec les maisons et les appartements sur une longue période; notre objectif était de comparer l'approche de collecte de données standardisée par rapport à celle reposant sur le souvenir. Les estimations absolues de collisions et les différences relatives ont été comparées entre les divers types d'habitation dans les deux études et nous avons trouvé des différences considérables dans les valeurs absolues de collisions, mais des différences relatives similaires dans les taux de collisions entre les deux types d'habitation. Les taux de collisions fondés sur le souvenir des citoyens dans notre étude (56,5\%) étaient très semblables à ceux de l'étude de 2012, dans laquelle 50,5\% des participants se souvenaient de la collision d'un oiseau avec une fenêtre à un moment donné dans le passé. Toutefois, les estimations de mortalité étaient 1,4 fois plus élevées dans l'étude de 2012 que dans notre étude fondée sur des recherches standardisées. Les maisons en milieu rural qui avaient un poste d'alimentation d'oiseaux avaient systématiquement les plus haut taux de collisions. Nos résultats montrent que les relevés faisant appel au souvenir peuvent être un outil utile pour comprendre l'importance relative des différents facteurs de risque causant les collisions d'oiseaux avec les fenêtres.

Key Words: bird mortality; bird-window collisions; buildings; citizen science; glass

\section{INTRODUCTION}

In Canada, an estimated 16-42 million birds die each year from collisions with windows (Machtans et al. 2013). This estimate equals close to one bird killed per person in Canada every year. Because many people can remember an instance where a bird collided with a window of their house (Bayne et al. 2012), this is an environmental issue that resonates with people when they are informed of it. However, a large percentage of the population is unaware of the magnitude of the issue and the need for more research to properly estimate and mitigate the impacts of birdwindow collisions (Arnold and Zink 2011, Sushinsky et al. 2013).
The field of citizen science, a form of public participation in scientific research, has grown in recent years as tools have become more available for dispersing information about projects and gathering data from the public (Bonney et al. 2014, Crain et al. 2014, Loss et al. 2015, Wiggins and Crowston 2015). The largest and some of the most successful citizen-science programs, including eBird, the Christmas Bird Count, Project Feeder Watch, and the Breeding Bird Survey, are in the field of ornithology (Tulloch et al. 2013). Recently, citizen-science projects have started collecting data on bird-window collisions. The majority of these bird-window collision projects have focused on tall buildings in the downtown core of large cities (Loss et al. 2015).

Address of Correspondent: Justine A Kummer, Department of Biological Sciences, University of Alberta, CW 405 Biological Sciences Building, Edmonton, AB, CANADA T6G 2E9, kummer@ualberta.ca 
Often overlooked are the effects of windows in houses. Houses likely represent a much greater potential threat to birds than tall commercial buildings because there are many more houses on the landscape. An earlier study by Machtans et al. (2013) estimated that houses were responsible for $90 \%$ of all bird-window collision mortalities in Canada.

The first attempt at using citizen science to study bird-window collisions at houses was conducted by Bayne et al. (2012). The project consisted of an online survey where homeowners were asked to recall whether they could remember a bird-window collision occurring at their home at any point in the past as well as more detailed questions about the number of collisions they remembered occurring in the previous year. This has been cited as the most extensive study to date on the effects of bird-window collisions at houses, and the results had a strong influence on recent estimates of Canadian and United States bird mortality caused by collisions with windows (Loss et al. 2014, Machtans et al. 2013).

The development of national estimates of mortality caused by bird-window collisions was quite successful in garnering media attention and raising public awareness about bird-window collisions (Machtans and Thogmartin 2014). However, the interest was primarily in the total number killed. The caveats and criticisms of the underlying data these estimates were based on were not discussed in the media despite being emphasized in the original papers (Best 2008, Machtans and Thogmartin 2014). For example, the data collected by Bayne et al. (2012) relied entirely on participant memory. This design may have led to recollection errors if respondents were unable to recall past collisions or if they recalled collisions that did not occur (Gaskell et al. 2000, Iarossi 2006). Participants also self-identified, potentially creating nonresponse errors or responses dominated by those who had observed a collision (Loss et al. 2012). Those participants with an interest in birds and a pre-established window collision issue may have been more likely to answer a series of questions relating to bird-window collisions than those who had not previously observed such an event. Such biases could result in collision and mortality estimates that are much higher than what actually occurs.

Here, we build upon the work of Bayne et al. (2012) by applying their recommendations for future research and more detailed monitoring. Specifically, we designed a citizen-science program to actively search for collision evidence at houses and apartments for an extended period of time, as well as evaluating what people remembered about collisions at their home. The objective was to see how a more standardized approach to citizen-science data collection influenced bird-window collision estimates and to see if the same patterns observed by Bayne et al. (2012) were observed using different methods of data collection.

\section{METHODS}

\section{Data collection protocol}

The Birds and Windows project (http://birdswindows.biology. ualberta.ca) was initiated in 2013 with two main components. First, we conducted a survey similar to that of Bayne et al. (2012) where people were asked to recall previous bird-window collisions. This was done to determine whether there were consistent patterns in people's recollection of collisions between the two studies. This survey was then used to recruit people to participate in systematic monitoring of their homes; participants were asked to search the perimeter of their residence for evidence of a bird-window collision on a daily basis.

When doing standardized searches, participants were asked to look for forms of collision evidence including dead or injured birds, and/or body smudges, feathers, or blood on windows. As well, if a participant saw or heard a collision occur outside of a perimeter search, it was included. Participants were asked to record every day that they searched for evidence. This was done to account for searcher effort and to ensure that days with no collisions were recorded. In completing searches, homeowners were asked to search within a $2-\mathrm{m}$ perimeter of their residence. Thus, birds that collided with a window, flew off, and died elsewhere might not have been detected. Participants were asked to look on the ground, in and around vegetation, and on balconies and sidewalks. Also, all windows were to be checked for evidence of a collision. To reduce the chance of evidence being missed, a pace of one step per second was recommended. After searching the residence once, participants were asked to reverse their direction and walk around a second time.

Homeowners living in apartments were also encouraged to participate. These participants were expected to walk the perimeter of their entire building and check the balconies of their own unit. Homeowners living in homes attached to at least one other dwelling (row housing, duplexes, semidetached and single attached homes) were required to search the perimeter and exterior walls of their individual unit. To maintain the privacy of other homeowners, participants were not to search the entire complex. Additionally, detached garages were to be monitored. The building classes used were based on the types of households provided by Statistics Canada (2016).

\section{Birds and Windows website}

Upon registering for the Birds and Windows project, homeowners completed a short survey on the past bird-window collision history at their home. Information was also collected on house and yard characteristics. The primary questions asked were the following: (1) How did you hear about the project? (2) What is your address? (3) What type of building do you live in (single detached homes, apartments, or residences attached to at least one other dwelling (row housing, duplexes, semidetached and single attached homes)? (4) Do you ever remember observing a bird-window collision in this residence? (5) Do you remember observing a bird-window collision in the past year at this residence? (6) Do you have a bird feeder within $10 \mathrm{~m}$ of your home? Following completion of the recall survey, participants were directed to the collision evidence search protocol and an onscreen calendar to be used in tracking the days they searched for evidence from the day of registration forward. For each day the homeowner searched their building, they were asked to enter evidence of whether or not a collision was found into the calendar. We assumed that homeowners walked around their residence and searched once per day. If no collision evidence was observed, there were no additional questions. When a collision was reported, several questions were asked. In this paper, we only used information from this question: Did the bird survive the initial collision? Based on the definitions outlined by Statistics Canada 
(2011) and using the addresses and/or postal codes provided by homeowners, Google Earth Pro was used to determine whether the location of each home was in an urban or rural setting. Homeowners were asked to email or upload photos of collision evidence to the Birds and Windows website to allow confirmation of each collision event and to identify species.

\section{Survey distribution}

The Birds and Windows website was launched in September 2013, and data were collected through May 2015. Requests to participate in the survey were distributed using multiple approaches. In promoting the project, posters and pamphlets were distributed throughout neighborhoods in Edmonton and outlying areas. Across Alberta, the project was presented to local bird stores, nature and bird groups, and small newspapers. Social media sites, including Facebook and Twitter, were set up, allowing the project to be accessible to a larger audience. Various universities and established organizations that promote citizenscience programs relating to bird conservation across North America were contacted electronically. Additionally, the Conservation Biology class at the University of Alberta participated for class credit during the fall 2013 and 2014 semesters. Four radio interviews were conducted with Canadian Broadcasting Corporation (CBC) Radio to promote the project. An additional $\mathrm{CBC}$ News article was published, and a segment aired on CBC Alberta Late Night News. A number of scientific outreach opportunities were taken to promote the project to children and the general public. Through personal contact with Environment Canada, Nature Alberta, the Alberta Conservation Association, the Alberta Biodiversity Monitoring Institute, and the Alberta Chapter of the Wildlife Society, the Birds and Windows project reached a number of potential participants and organizations with an established interest in bird conservation and citizen science. Registered participants who had stopped searching for evidence were contacted again after a few months to encourage them to continue. In addition, in registering with the survey, participants had the option of requesting a weekly email reminding them to participate.

\section{Data analysis}

All homeowner observations were checked for consistency. Confirmation emails were sent to participants with suspicious entries, e.g., 30 observations entered at once, multiple collisions entered in 1 day, and collisions entered for the time period before the participant had initially signed up for the project. If confirmation of these observations was not provided, they were excluded from our analysis.

Each residence was divided into one of the five major residence classes outlined by Bayne et al. (2012): (1) rural residences with a bird feeder, (2) rural residences without a bird feeder, (3) urban residences with a bird feeder, (4) urban residences without a bird feeder, and (5) apartments and condo complexes. Residences in the other building class (row housing, duplexes, semidetached and single attached homes) were grouped with single attached houses.

Five response variables were calculated for each homeowner: (1) CollisionEver was derived from the recall-survey question, "Do you ever remember observing a bird-window collision in this residence?" (2) Collision Year was derived from the recall-survey question, "Do you remember observing a bird-window collision in the past year at this residence?" (3) CollisionSearch referred to whether or not a collision was observed during the homeowner's standardized searches. (4) CollisionNumber was the predicted number of collisions at each residence in one year based on standardized search data. (5) FatalityNumber was the predicted number of collisions that resulted in a fatality in one year based on standardized search data.

Because not all participants collected data for the same length of time, we had to adjust for differential effort when estimating CollisionNumber and FatalityNumber. Two options were explored. First, we calculated the proportion of the year a person reported collecting data by taking the total number of days of observations and dividing by 365 . Second, we evaluated the proportion of the year a person participated by taking the numbers of days between when they registered and the last date when they entered data and dividing by 365 . These proportions were natural logarithm-transformed and then applied as either a statistical offset or fixed effect in negative binomial regression models for CollisionNumber and FatalityNumber. Negative binomial regression was used because of overdispersion in the raw count data relative to a Poisson distribution. Fit between these two approaches to correct for sampling effort was compared via Akaike Information Criteria (AIC; Burnham and Anderson 2004), and the approach that provided the best model fit for both CollisionNumber and FatalityNumber was chosen when reporting final results. Both proportion-of-the-year approaches modeled count data as a rate that allowed us to report CollisionNumber and FatalityNumber on an annual basis. All results are presented as mean probabilities and counts. Models were created in STATA 13 (StataCorp, College Station, Texas, USA, http://www.stata.com/). Only homeowners who completed a minimum of 1 month of observations (28 days) outlined in the protocol were included in these analyses.

\section{RESULTS}

\section{Participant recruitment}

A total of 1315 participants registered with the project. Of those, 981 homeowners completed bird-window collision observations. The 2 most successful survey distribution methods were word of mouth (474 homeowners recruited) and email (353 homeowners recruited). A total of 161 people heard about the project through students in the Biology 367 Conservation Biology class, 104 participants heard about the project through pamphlet distribution, and 99 through the Birds and Windows Facebook and Twitter accounts. Another 57 people first heard about the project through media outreach, including newspaper articles and radio and TV interviews.

Among the participants, 1226 were from Canada, 56 were from the United States, and 8 were from the United Kingdom. There were additional participants from Australia (2 homeowners), India (1 homeowner), and various European countries, including the Czech Republic (1 homeowner), Ireland (1 homeowner), Netherlands (1 homeowner), Norway (1 homeowner), and Poland (2 homeowners). For 16 homeowners, their country was unknown. In Canada, 995 homeowners from Alberta registered with the project. There were registered homeowners from all Canadian provinces, with the next 2 highest being British Columbia (101 homeowners) and Ontario (56 homeowners). 
Table 1. Akaike information criterion (AIC) scores and collision and fatality estimates when correcting for participant effort. Summary also includes the relative difference between models and the best model ( $\triangle \mathrm{AIC}$ ), Akaike weights (AICw), log-likelihood ( $L$ ), and number of parameters $(\mathrm{K})$.

\begin{tabular}{|c|c|c|c|c|c|c|}
\hline & Estimate & AIC & $\Delta \mathrm{AIC}$ & $\mathrm{AICw}$ & $L$ & $\mathrm{~K}$ \\
\hline \multicolumn{7}{|l|}{ Fatality } \\
\hline \multicolumn{7}{|l|}{ Offset } \\
\hline Proportion of year with participation & 0.48 & 278.22 & 0.00 & 0.74 & -137.11 & 2 \\
\hline Proportion of year where data collected & 0.68 & 280.32 & 2.10 & 0.26 & -138.16 & 2 \\
\hline \multicolumn{7}{|l|}{ Fixed effect } \\
\hline Proportion of year with participation & 1.11 & 279.61 & 0.00 & 0.56 & -136.81 & 3 \\
\hline Proportion of year where data collected & 0.57 & 280.09 & 0.48 & 0.44 & -137.04 & 3 \\
\hline \multicolumn{7}{|l|}{ Collision } \\
\hline \multicolumn{7}{|l|}{ Offset } \\
\hline Proportion of year with participation & 5.55 & 1105.82 & 0.00 & 1.00 & -550.91 & 2 \\
\hline $\begin{array}{l}\text { Proportion of year where data collected } \\
\text { Fixed effect }\end{array}$ & 7.95 & 1130.84 & 25.02 & 0.00 & -563.42 & 2 \\
\hline Proportion of year with participation & 9.88 & 1107.63 & 0.00 & 1.00 & -550.82 & 3 \\
\hline Proportion of year where data collected & 5.89 & 1131.84 & 24.21 & 0.00 & -562.92 & 3 \\
\hline
\end{tabular}

Because we did not have a representative sample for all of Canada, only homeowners from Alberta were included in this analysis. Of the 995 registered participants from Alberta, 768 homeowners submitted observations. Figure 1 shows the number of participants by the nearest city or town in Alberta.

Fig. 1. Map of the province of Alberta, showing location of participants. Size of symbol indicates the number of participants in that community.

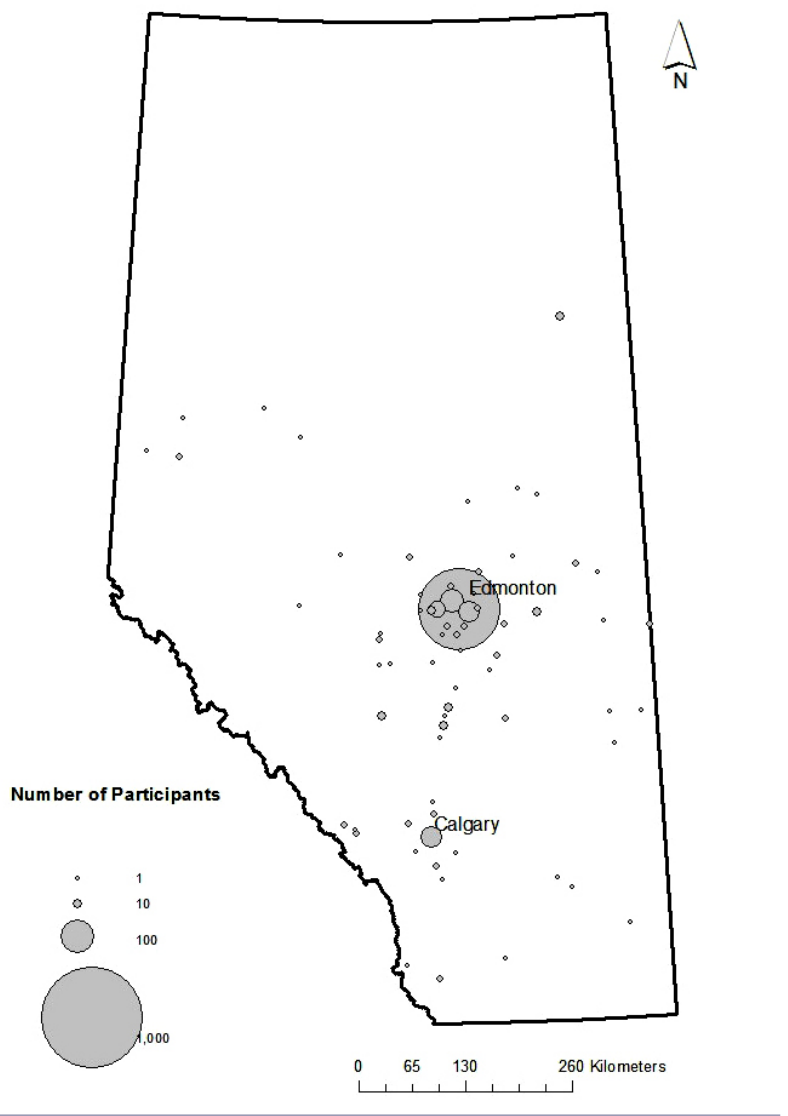

After entering one observation $12.2 \%$ of homeowners did not continue with the project. A total of 381 participants reached the minimum 28 days of standardized searches required by the project protocol. The average length of participation was 44 (SD 76) days. The top 5 numbers of entries were $610,554,527,526$, and 511 days.

\section{Recollection of past bird-window collisions from survey questions}

Upon registering for the Birds and Windows project, participants were asked about collisions that they remembered occurring in the past. CollisionEver was $56.5 \%$ for participants in Alberta who participated for at least one month, while CollisionYear was $43.8 \%$.

The rank order for CollisionYear using the 5 main residence classes was rural residence with a feeder $(95.7 \%$ of homeowners reported a collision), urban residence with a feeder $(56.2 \%)$, rural residence without a feeder $(53.8 \%)$, urban residence without a feeder $(36.9 \%)$, and apartments $(11.6 \%)$. Overall, $70 \%$ of the homeowners who reported a collision in the previous year at their home observed a collision while conducting the standardized searches around their home.

\section{Observed collisions from standardized searches}

Based on AIC, model fit was better when we used the proportion of the year a person participated in the project rather than the proportion of the year they made actual observations $(\triangle \mathrm{AIC}=$ 25.02). Treating this variable as an offset with the coefficient set to 1 provided a better fit than a fixed-effect model $(\triangle \mathrm{AIC}=1.81)$. All of the results are reported using this approach to correct for differential effort (Table 1).

Alberta homeowners entered 34,114 observations in the Birds and Windows project database. Of these observations, 930 collisions and 102 fatalities were recorded. Of these, 76 collisions were verified through photos. Among homeowners who participated for at least 1 month, $42.3 \%$ entered a collision during the standardized searches. The top 5 residences reported $68,44,36$, 
30, and 27 collisions and 9, 9, 4, 4, and 3 fatalities, respectively. Based on our model, correcting for differential sampling effort, we found the mean number of collisions occurring annually to be $5.55(95 \%$ confidence interval $[\mathrm{CI}]=4.61-6.70)$, whereas fatalities per year were estimated to be $0.48(95 \% \mathrm{CI}=0.32-0.72)$. These estimates are based on data collected from all residence types and pooled together (Table 2).

Table 2. Comparative statistics between survey questions and standardized searches from the Birds and Windows project and the results presented in Bayne et al. (2012). ${ }^{\dagger}$

\begin{tabular}{lcc}
\hline \hline & Birds and Windows & Bayne et al. (2012) \\
\hline Participants & 381 & 1458 \\
CollisionEver & $56.5 \%$ & $50.5 \%$ \\
CollisionYear & $43.9 \%$ & $39.0 \%$ \\
CollisionSearch & $42.3 \%$ & $\sim$ \\
CollisionNumber & 5.55 & 1.7 \\
FatalityNumber & 0.48 & 0.7 \\
& & \\
\hline
\end{tabular}

${ }^{\dagger}$ The five response variables included (1) CollisionEver, the probability of a participant reporting a bird-window collision at any point since they moved into their current residence; (2) Collision Year, the probability of a participant reporting a bird-window collision within the year prior to beginning the survey; (3) CollisionSearch, the probability of a participant reporting a bird-window collision during the homeowner's standardized searches; (4) CollisionNumber, the predicted number of collisions at each residence in one year based on standardized search data; and (5) FatalityNumber, the predicted number of collisions that resulted in a fatality in one year based on standardized search data. The results from the Birds and Windows project are presented as mean probabilities and counts. Only homeowners from Alberta who completed the 1 month of observations ( 28 days) outlined in the protocol were included in analysis. The results presented from Bayne et al. (2012) were taken directly from that paper. As a result of different study designs, there is no result for CollisionSearch from Bayne et al. (2012).

Based on standardized searches, the rank order for CollisionSearch was rural residences with a feeder $(91.7 \%$ probability of a collision being observed during standardized searches), rural residences without a feeder $(69.2 \%)$, urban residences with a feeder $(50.5 \%)$, urban residences without a feeder $(33.9 \%)$, and apartments $(25.0 \%)$. CollisionNumber followed the same pattern (Table 3). The pattern for FatalityNumber was similar apart from an unexpectedly low number of fatalities at rural houses without feeders. Except for the fatality rate at these houses, apartments had the lowest number of collisions and lower fatality rates than the other residence classes (Table 3).

Rural residences with a bird feeder were predicted to have 7.7 times more collisions than apartments and 3.7 times more than urban residences without a bird feeder. Combining all building classes, rural residences were predicted to have 3.4 times more collisions than urban residences, and residences with a bird feeder were predicted to have 1.9 times more collisions than residences without a bird feeder.

\section{DISCUSSION}

\section{Are surveys based on recall of past events comparable to standardized searches?}

We compared the results from our standardized searches, our recall-survey data, and the recall-survey data reported by Bayne et al. (2012). This comparison revealed large differences in absolute values of collisions but similar relative rankings between residence types.

Four collision metrics reported by Bayne et al. (2012) could be compared with our study (Table 3). Comparing CollisionEver and CollisionYear, which used the same questions and methods as Bayne et al. (2012), we found very similar results. Overall, 6.5\% more of our participants remembered a bird colliding with a window at some time in the past compared with Bayne et al. (2012). Within the previous year, $4.9 \%$ more people in our group remembered a collision compared with the study by Bayne et al. (2012). Overlapping binomial CIs for both studies indicate no significant difference between studies when the same techniques were used. This suggests that potential biases that may have existed in the Bayne et al. (2012) study were similar to those in our recall survey. If survey methods based on past recall were a completely unreliable way of determining the likelihood of a birdwindow collision occurring, we would have expected high variability with inconsistent patterns over the four years of surveys.

The relative ranking of the five types of residences was also generally consistent when systematic surveys and past recall were compared. The relative ranking for CollisionEver between the five types of residences was identical between studies. CollisionYear was similar to the result reported by Bayne et al. (2012), except for one year during which participants at rural residences without a bird feeder had a higher probability of remembering a collision than those at rural residences with a bird feeder (Table 3). In our study, rural residences with a bird feeder always had the highest collision rate regardless of whether the data were collected based on past recall or standardized searches. Absolute values did vary between the two studies for the same metrics, although in most cases the 95\% CIs overlapped (Table 3). These results are additionally comparable with those of an experiment where we manipulated the presence and distance of a bird feeder from a window at houses (Kummer and Bayne 2015). Throughout that study bird feeder presence was shown to increase collision rate 1.57-2.20 times.

The similar relative ranking of residence types in our study and that of Bayne et al. (2012) increases confidence that these patterns are robust. However, there were differences in absolute values. We suggest participants in the standardized searches were more likely to take note of things like body smudges, collision noises, and feathers or blood on the window once they were told these were evidence of a bird-window collision. When participants were asked survey questions, this type of evidence was likely more difficult to recall than finding a dead or injured bird and may explain the differences.

Fatality estimates were 1.4 times higher in Bayne et al. (2012) than in our study, which is somewhat surprising. Also, the relative ranking for fatality rate was somewhat different between residence 
Table 3. Rates of bird-window collisions and fatalities reported and predicted from survey questions and standardized searches from the Birds and Windows project for five residence types in the province of Alberta, Canada compared with the previous rates predicted for the years 2009 and 2010 in Bayne et al. (2012). Numbers in parentheses are the lower and upper bounds of $95 \%$ confidence intervals. $\dagger$

\begin{tabular}{|c|c|c|c|c|c|}
\hline Response Variable & Apartment & Urban, no feeder & Urban, with feeder & Rural, no feeder & Rural, with feeder \\
\hline $\begin{array}{l}\text { CollisionEver } \\
\text { Birds and Windows } \\
\text { Bayne et al. (2012) }\end{array}$ & $0.09(0.01-0.18)$ & $0.50(0.43-0.58)$ & $0.72(0.63-0.81)$ & $0.92(0.76-1.00)$ & $1.00(1.00-1.00)$ \\
\hline $\begin{array}{l}2009 \\
2010\end{array}$ & $\begin{array}{l}0.27(0.14-0.39) \\
0.20(0.10-0.30)\end{array}$ & $\begin{array}{l}0.59(0.53-0.64) \\
0.45(0.39-0.51)\end{array}$ & $\begin{array}{l}0.70(0.63-0.77) \\
0.71(0.65-0.77)\end{array}$ & $\begin{array}{l}0.82(0.62-1.00) \\
0.88(0.77-0.99)\end{array}$ & $\begin{array}{l}0.86(0.68-1.00) \\
0.93(0.85-1.00)\end{array}$ \\
\hline Collision Year & & & & & \\
\hline $\begin{array}{l}\text { Birds and Windows } \\
\text { Bayne et al. (2012) }\end{array}$ & $0.12(0.02-0.22)$ & $0.37(0.30-0.44)$ & $0.56(0.47-0.66)$ & $0.54(0.22-0.85)$ & $0.96(0.87-1.00)$ \\
\hline $\begin{array}{l}2009 \\
2010\end{array}$ & $\begin{array}{l}0.20(0.10-0.30) \\
0.13(0.06-0.20)\end{array}$ & $\begin{array}{l}0.37(0.32-0.42) \\
0.25(0.20-0.29)\end{array}$ & $\begin{array}{l}0.53(0.46-0.60) \\
0.44(0.38-0.51)\end{array}$ & $\begin{array}{l}0.61(0.38-0.84) \\
0.84(0.70-0.97)\end{array}$ & $\begin{array}{l}0.68(0.45-0.91) \\
0.67(0.54-0.81)\end{array}$ \\
\hline CollisionSearch & & & & & \\
\hline $\begin{array}{l}\text { Birds and Windows } \\
\text { CollisionNumber }\end{array}$ & $0.25(0.12-0.38)$ & $0.34(0.27-0.41)$ & $0.50(0.41-0.60)$ & $0.69(0.40-0.98)$ & $0.92(0.80-1.00)$ \\
\hline $\begin{array}{l}\text { Birds and Windows } \\
\text { Bayne et al. (2012) }\end{array}$ & $2.57(1.36-4.85)$ & $4.25(1.13-15.98)$ & $5.40(1.41-20.73)$ & $5.79(0.99-33.89)$ & $19.7(4.37-88.81)$ \\
\hline $\begin{array}{l}2009 \\
2010\end{array}$ & $\begin{array}{l}0.85(0.45-1.26) \\
0.22(0.09-0.35)\end{array}$ & $\begin{array}{l}1.11(0.87-1.35) \\
0.69(0.53-0.85)\end{array}$ & $\begin{array}{l}2.03(1.49-2.56) \\
1.81(1.37-2.25)\end{array}$ & $\begin{array}{l}5.87(0.93-10.8) \\
5.10(1.76-8.44)\end{array}$ & $\begin{array}{l}3.67(0.54-6.81) \\
4.29(2.17-6.43)\end{array}$ \\
\hline FatalityNumber & & & & & \\
\hline $\begin{array}{l}\text { Birds and Windows } \\
\text { Bayne et al. (2012) }\end{array}$ & $0.31(0.07-1.43)$ & $0.33(0.01-8.34)$ & $0.44(0.02-10.93)$ & $0.24(0.01-20.0)$ & $1.82(0.06-55.2)$ \\
\hline 2009 & $0.22(0.06-0.37)$ & $0.50(0.36-0.64)$ & $0.65(0.43-0.87)$ & $1.62(0.00-3.29)$ & $2.16(0.00-4.37)$ \\
\hline 2010 & $0.06(0.01-0.13)$ & $0.20(0.13-0.26)$ & $0.71(0.49-0.93)$ & $2.12(0.44-3.81)$ & $1.38(0.54-2.24)$ \\
\hline $\begin{array}{l}\text { Sample size } \\
\text { Birds and Windows }\end{array}$ & & & & & \\
\hline $\begin{array}{l}\text { CollisionEver } \\
\text { CollisionYear } \\
\text { CollisionSearch, } \\
\text { CollisionNumber, and } \\
\text { FatalityNumber } \\
\text { Bayne et al. (2012) }\end{array}$ & $\begin{array}{l}\mathrm{n}=43 \\
\mathrm{n}=43 \\
\mathrm{n}=48\end{array}$ & $\begin{array}{l}\mathrm{n}=177 \\
\mathrm{n}=176 \\
\mathrm{n}=189\end{array}$ & $\begin{array}{l}\mathrm{n}=104 \\
\mathrm{n}=105 \\
\mathrm{n}=107\end{array}$ & $\begin{array}{l}\mathrm{n}=13 \\
\mathrm{n}=13 \\
\mathrm{n}=13\end{array}$ & $\begin{array}{l}\mathrm{n}=24 \\
\mathrm{n}=23 \\
\mathrm{n}=24\end{array}$ \\
\hline $\begin{array}{l}2009 \\
2010\end{array}$ & $\begin{array}{l}\mathrm{n}=117 \\
\mathrm{n}=173\end{array}$ & $\begin{array}{l}n=404 \\
n=433\end{array}$ & $\begin{array}{l}\mathrm{n}=219 \\
\mathrm{n}=272\end{array}$ & $\begin{array}{l}\mathrm{n}=22 \\
\mathrm{n}=35\end{array}$ & $\begin{array}{l}\mathrm{n}=18 \\
\mathrm{n}=54\end{array}$ \\
\hline $\begin{array}{l}{ }^{\dagger} \text { The five response varia } \\
\text { moved into their curren } \\
\text { beginning the survey; } \\
\text { searches; ( } 4 \text { ) Collision } \\
\text { FatalityNumber, the pr } \\
\text { Birds and Windows pro } \\
\text { observations ( } 28 \text { days) } \\
\text { that paper. As a result }\end{array}$ & $\begin{array}{l}\text { uded (1) Collisio } \\
\text { ice; (2) Collision } \\
\text { onSearch, the pro } \\
\text { the predicted nun } \\
\text { umber of collisio } \\
\text { 15) are presented } \\
\text { in the protocol w }\end{array}$ & $\begin{array}{l}\text { the probability of } \\
\text { e probability of a } \\
\text { ty of a participant } \\
\text { collisions at each } \\
\text { tresulted in a fatal } \\
\text { n probabilities and } \\
\text { luded in analysis. } \\
\text { s no result for Coll }\end{array}$ & $\begin{array}{l}\text { icipant reporting a bi } \\
\text { ipant reporting a bird } \\
\text { ting a bird-window cc } \\
\text { nce in one year based } \\
\text { one year based on sta } \\
\text { ts. Only homeowners } \\
\text { sults presented from } \\
\text { Search from Bayne et }\end{array}$ & $\begin{array}{l}\text { indow collision at } \\
\text { dow collision with } \\
\text { on during the hom } \\
\text { tandardized searc } \\
\text { dized search data } \\
\text { Alberta who con } \\
\text { e et al. (2012) were } \\
012) \text {. }\end{array}$ & $\begin{array}{l}\text { point since they } \\
\text { ee year prior to } \\
\text { ner's standardized } \\
\text { ta; and (5) } \\
\text { results from the } \\
\text { ed the } 1 \text { month of } \\
\text { en directly from }\end{array}$ \\
\hline
\end{tabular}

types and across studies. This was not unexpected because there were a small number of reported fatalities in the standardized searches and a small sample size for rural residences without feeders. This resulted in wide CIs and a very low number of fatalities estimated to occur at rural residences without a feeder. Perhaps more important is the fact that the magnitude of difference in fatalities was influenced by the approach used to adjust for differential sampling effort. The maximum fatality rate was 2.3 times greater than the minimum rate $(1.11$ fatalities to 0.48 fatalities) depending on method of correction. Importantly, the fatality estimate from Bayne et al. (2012) fell in the middle of this range, suggesting that past efforts to estimate the total number of birds killed by window collisions is starting from a reasonable baseline.

\section{Improving citizen science}

That most of the metrics used in our study and that of Bayne et al. (2012) were similar in the relative ranking between residence types suggests that recall surveys may be a useful tool for understanding the relative importance of different risk factors causing bird-window collisions. Estimating absolute collision rates remains challenging, however. Whether to correct for differential effort using a statistical approach versus only using data from observers who participate for a fixed number of days is a good example of the challenges involved in getting a precise and accurate estimate of collisions and mortality, even with systematic surveys.

When determining the best way to deal with variation in participant effort, we tried a number of different approaches. In 
using the proportion of the year a homeowner participated, we were able to account for gaps in their observations. Although homeowners were asked to participate for a period of 1 month, there were a number of instances when a homeowner left gaps between observations, ranging in length from 1 day to a couple of months. In using this approach to determine our collision and fatality estimates, we suspect the homeowner monitored their house for bird-window collisions during these gaps and simply forgot to enter the days when they did not observe a collision, or the homeowner remained aware of the project over this time but did not conduct official searches around their home. These gaps have been included in calculating the proportion of the year the homeowner participated in the project.

We believe reducing sources of potential error will be difficult using citizen science, and uncertainty in absolute collision and mortality rates will likely persist using such an approach to data collection. However, there remain a number of benefits for conducting similar studies across Canada, including identifying species-specific, seasonal, and geographic trends. As well, only by having a more accurate estimate and representation of collisions across the country will steps be taken at the necessary levels to reduce the number of collisions occurring. Recognizing that the need for more detailed information exists should not diminish additional progress in dealing with the issue. We suggest logical next questions to answer are the following: (1) What role can citizen scientists play in helping determine how to reduce birdwindow collisions? (2) Are relative comparisons sufficient to understand the efficacy of different mitigation options? (3) Is past recall sufficiently accurate that we can understand the drivers that make one house more likely to incur collisions than another when seeking mitigation solutions? (4) How important is it to conduct such studies in other cities?

One area in which we predict that citizen scientists will be very effective in reducing bird-window collisions is in testing the efficacy of mitigation options. Although there have been a number of successful localized studies as new mitigation options are developed ( BirdTape, http://www.abcbirdtape.org/; Ornilux Bird Protection Glass, http://www.ornilux.com; Feather Friendly Bird Deterrent Window Films, http://www.conveniencegroup.com/ featherfriendly/feather-friendly; Window Alert, http://windowalert. com), these products have yet to be compared in the same study at actual houses to determine efficacy. Our recent bird feeder study used citizen scientists to look at the effect of bird feeder presence and distance at houses (Kummer and Bayne 2015). This experiment did not require we know the absolute reduction in bird-window collisions, and until a technological solution to record absolute numbers of collisions is possible we argue that using citizen scientists to collect data that provide relative comparisons between various mitigation options is the most effective option for acquiring this information in real-world scenarios.

Our intention when we originally started this project was to obtain estimates of bird-window collisions from an area larger than Alberta. We spent considerable time trying to elicit other universities and agencies to participate with limited success. If we desire national estimates of bird-window collisions, then a far greater number of cities and homes would have to be sampled, because the Edmonton area is not representative of all of Canada.
The participation of undergraduate classes in both our study and that of Bayne et al. (2012) shows how approximately 360 students over 4 years helped recruit more than 3000 participants for a recall study. This model should be encouraged across Canada for two reasons. First, only by having dedicated personnel in each city is participation likely to occur because personal relationships play a large roll in getting homeowners to participate. Second, the simple act of teaching students about bird-window collisions, who then inform their friends and families, increases awareness about the issue and, in our experience, encourages people to try different mitigation options. A centralized agency that is able to provide resources to advertise, encourage participation through some type of reward system, and provide the data collection materials and data portal is sorely needed.

Overall, we suggest that our research and that of Bayne et al. (2012) demonstrate that bird-window collisions at houses are an issue in the Edmonton area. We were able to observe the same bird-window collision patterns as Bayne et al. (2012) using both the same and different methods of data collection. Regardless of how data were collected, these studies indicate that a large number of birds are colliding with and dying at windows. Although there are benefits in developing new national estimates, we suggest shifting our scientific objective from estimating the magnitude of collisions to using citizen scientists to test and implement mitigations for bird-window collisions and thereby focus future research and conservation effort.

Responses to this article can be read online at: http://www.ace-eco.org/issues/responses.php/820

\section{Acknowledgments:}

We want to thank the 1315 homeowners who registered and participated in the Birds and Windows project. Their continued dedication in searching for bird-window collision evidence every day was greatly appreciated. This project would not have been a success without them. We also want to thank C. Charchuk, K. Garbrah, E. Grinde, J. Hyun-Joo Ko, E. Jutras, A. Menzies, C. Scobie, and C. Tse. The Department of Biological Sciences at the University of Alberta and Environment Canada provided funding for the project. This research was conducted under permit \#Pro00041311 from the Research Ethics Board at the University of Alberta.

\section{LITERATURE CITED}

Arnold, T. W., and R. M. Zink. 2011. Collision mortality has no discernible effect on population trends of North American birds. PLoS ONE 6(9):e24708. http://dx.doi.org/10.1371/journal. pone. 0024708

Bayne, E. M., C. A. Scobie, and M. Rawson-Clark. 2012. Factors influencing the annual risk of bird-window collisions at residential structures in Alberta, Canada. Wildlife Research 39:583-592. http://dx.doi.org/10.1071/WR11179

Best, J. 2008. Birds - dead and deadly: why numeracy needs to address social construction. Numeracy 1(1):6. http://dx.doi. org/10.5038/1936-4660.1.1.6 
Bonney, R., J. L. Shirk, T. B. Phillips, A. Wiggins, H. L. Ballard, A. J. Miller-Rushing, and J. K. Parrish. 2014. Next steps for citizen science. Science 343:1436-1437. http://dx.doi.org/10.1126/ science. 1251554

Burnham, K. P., and D. R. Anderson. 2004. Multimodel inference - understanding AIC and BIC in model selection. Sociological Methods \& Research 33:261-304. http://dx.doi.org/10.1177/0049124104268644

Crain, R., C. Cooper, and J. L. Dickinson. 2014. Citizen science: a tool for integrating studies of human and natural systems. Annual Review of Environment and Resources 39:641-665. http:// dx.doi.org/10.1146/annurev-environ-030713-154609

Dunn, E. H. 1993. Bird mortality from striking residential windows in winter. Journal of Field Ornithology 64:302-309.

Gaskell, G. D., D. B. Wright, and C. A. O'Muircheartaigh. 2000. Telescoping of landmark events: implications for survey research. Public Opinion Quarterly 64:77-89.

Iarossi, G. 2006. The power of survey design: a user's guide for managing surveys, interpreting results, and influencing respondents [electronic resource]. World Bank, Washington, D.C., USA. http://dx.doi.org/10.1596/978-0-8213-6392-8

Klem, D. 1990. Collisions between birds and windows: mortality and prevention. Journal of Field Ornithology 61(1):120-128.

Kummer, J. A., and E. M. Bayne. 2015. Bird feeders and their effect on bird-window collisions at residential houses. Avian Conservation and Ecology 10(2):6. http://dx.doi.org/10.5751/ ace-00787-100206

Loss, S. R., S. S. Loss, T. Will, and P. P. Marra. 2015. Linking place-based citizen science with large-scale conservation research: a case study of bird-building collisions and the role of professional scientists. Biological Conservation 184:439-445. http://dx.doi. org/10.1016/j.biocon.2015.02.023

Loss, S. R., T. Will, S. S. Loss, and P. P. Marra. 2014. Bird-building collisions in the United States: estimates of annual mortality and species vulnerability. Condor 116(1):8-23. http://dx.doi. org/10.1650/condor-13-090.1

Loss, S. R., T. Will, and P. P. Marra. 2012. Direct human-caused mortality of birds: improving quantification of magnitude and assessment of population impact. Frontiers in Ecology and the Environment 10(7):357-364. http://dx.doi.org/10.1890/110251

Machtans, C. S., and W. E. Thogmartin. 2014. Understanding the value of imperfect science from national estimates of bird mortality from window collisions. Condor 116(1):3-7. http://dx. doi.org/10.1650/condor-13-134.1

Machtans, C. S., C. H. R. Wedeles, and E. M. Bayne. 2013. A first estimate for Canada of the number of birds killed by colliding with building windows. Avian Conservation and Ecology 8(2):6. http://dx.doi.org/10.5751/ACE-00568-080206
Statistics Canada. 2011. From urban areas to population centresStatistics Canada, Ottawa, Ontario, Canada. [online] URL: http://www.statcan.gc.ca/eng/subjects/standard/sgc/notice/ sgc-06

Statistics Canada. 2016. Private households by structural type of dwelling, by province and territory (2011 census). Statistics Canada, Ottawa, Ontario, Canada.[online] URL: http://www. statcan.gc.ca/tables-tableaux/sum-som/101/cst01/deffamil55a-eng. htm?returnfile $=$ famil55a-eng.htm

Sushinsky, J. R., J. R. Rhodes, H. P. Possingham, T. K. Gill, and R. A. Fuller. 2013. How should we grow cities to minimize their biodiversity impacts? Global Change Biology 19:401-410. http:// dx.doi.org/10.1111/gcb.12055

Tulloch, A. I. T., H. P. Possingham, L. N. Joseph, J. Szabo, and T. G. Martin. 2013. Realising the full potential of citizen science monitoring programs. Biological Conservation 165:128-138. http://dx.doi.org/10.1016/j.biocon.2013.05.025

Wiggins, A., and K. Crowston. 2015. Surveying the citizen science landscape. First Monday 20(1):5. http://dx.doi.org/10.5210/fm. v20i1.5520
Editor-in-Chief: Ryan Norris Subject Editor: Scott Wilson
Sponsored by the Society of Canadian Ornithologists and Bird Studies Canada Parrainée par la Société des ornithologistes du Canada et Études d'oiseaux Canada

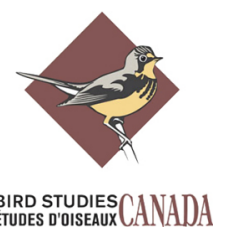

